\title{
Case Report of Metastatic Colon Adenocarcinoma with Laryngeal Deposits
}

\author{
Stella Garvie ${ }^{1, *}$, Polycarp Gana ${ }^{1}$, Jamal Uraiby ${ }^{2}$ \\ ${ }^{1}$ Kettering General Hospital Foundation Trust, Ear, Nose \& Throat Department, Kettering, NN16 8UZ, United Kingdom. \\ ${ }^{2}$ Kettering General Hospital Foundation Trust, Department of Cellular Pathology, Kettering, NN16 8UZ, United Kingdom.
}

\begin{abstract}
The larynx is known to be a very rare site for metastases of tumours of various regions due to its terminal position in the lymphatic circulation system. The last case of a massive transglottic deposit of the larynx with associated classical symptoms was described in 1997. Our case describes a unique clinical history of a laryngeal metastasis of colon adenocarcinoma without typical physical presentation but mimicking Paget's disease.
\end{abstract}

Due to its scarce appearance in clinical practice and medical literature, laryngeal metastasis of cancer can be an immense diagnostic challenge. The aforementioned case is a great contribution to the understanding and management of such patients.

Keywords: Adenocarcinoma, Cricoid, Laryngeal, Laryngoscopy, Metastases, Sclerosis.

\section{INTRODUCTION}

Metastases to the larynx are generally rare in clinical practice [1-3]. The explanation of that is based on the terminal location of this organ in the lymphatic-vascular circulation. Among the laryngeal metastases that are described in literature, the most common sources of malignancy are melanomas and renal carcinomas [4], leaving gastrointestinal tract, lung and breast cancer rather sporadic primary sources. In this background metastases of colon carcinoma to the larynx seem almost overly subtle. This makes any clinical case of laryngeal secondary deposits from a primary colon tumour a very valuable clinical and educational finding. In this article we report a case of laryngeal metastases of colorectal adenocarcinoma with atypical presentation that posed a diagnostic dilemma.

\section{CLINICAL CASE}

A 71-year old patient with known history of colorectal carcinoma with lung metastases was admitted to the hospital because of respiratory distress. By then he had already received 29 cycles of chemotherapy and was due to undergo the 30th one. Prior to admission he experienced a long-term shortness of breath with wheeze and productive cough which seemed not to improve after antibiotic therapy. He subsequently developed respiratory failure from an upper airway obstruction; intubation was attempted with multiple failures so an urgent tracheostomy was conducted under local anaesthetic.

Flexible nasoendoscopy showed significant narrowing of the subglottic airway, otherwise, examination of ear, nose and throat was unremarkable. CT and MRI scans of the neck were

*Address correspondence to this author at the Kettering General Hospital Foundation Trust, Ear, Nose \& Throat Department, Kettering, NN16 8UZ, United Kingdom. E-mail: stella.garvie@nhs.net performed which showed an infraglottic stenosis but no mass in the upper aerodigestive tract or cervical lymphadenopathy. However, there was evidence of cricoid cartilage thickening but the radiologist did not associate this finding with any possibility of malignant change (Figs. 1, 2).
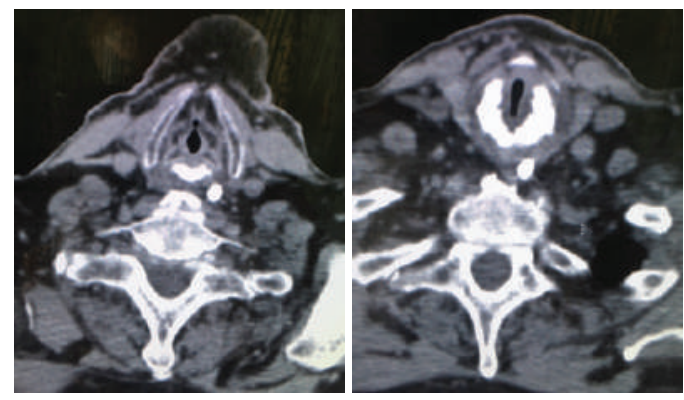

Fig. (1). CT Scan of the Neck of the Patient with Respiratory Distress.

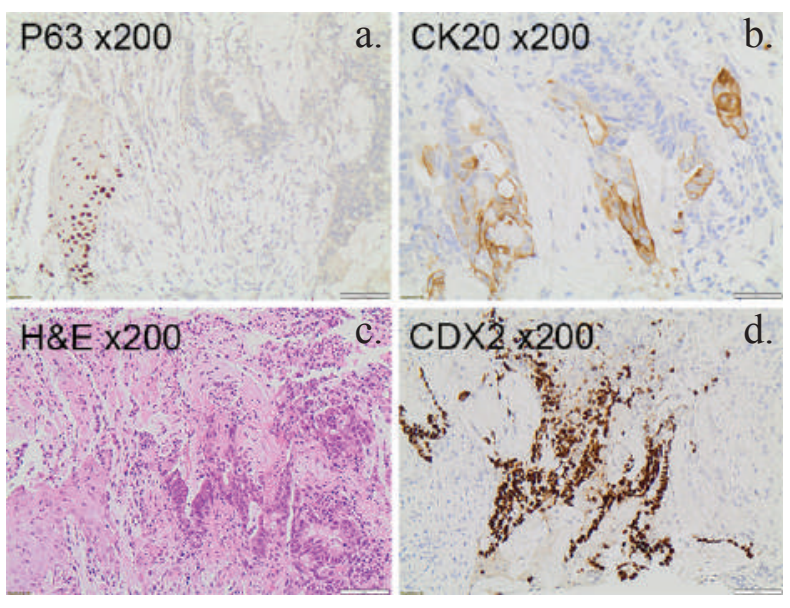

Fig. (2). Specimen with Metastatic Colorectal Carcinoma. (a) Staining P63, (b) CK20, (c) H\&E and (d) CDX2.

www.njhsciences.com 
The laryngeal airway although narrowed, was still adequate with no mucosal breaches but only thickening of the laryngeal structures and congested post cricoid and subglottic areas.

The case was discussed at the Head and Neck Multi-Disciplinary Team meeting and the official MDT comment and diagnosis was "extensive sclerosis of cricoid" consistent with Paget's disease. Direct laryngoscopy was performed and biopsy of the subglottic area was taken on insistence of the oncologist for histology. Unfortunately, the patient was deceased before the histopathology report.

\section{HISTOPATHOLOGICAL REPORT}

Most of the sample consisted of fragments of respiratory type mucosa showing non-specific chronic inflammation. However, one fragment showed surface ulceration and contained occasional disrupted glands lined by dysplastic epithelium with associated necrosis. Small fragments of squamous epithelium were also present.

Immunohistochemistry confirmed dysplastic glandular epithelium that was CDX2 positive, CK positive, CEA positive, CK negative, P63 negative and TTF-1 negative. This immunostain profile was in keeping with colorectal epithelial origin and consistent with metastatic colorectal carcinoma.

\section{DISCUSSION}

Majority of the tumours that metastasise to the larynx are either melanomas $(29 \%)$ or carcinomas, especially renal $(25 \%)$, gastrointestinal $(14 \%)$, lung $(9 \%)$, breast $(8 \%)$, and prostate (5\%) [3] but the overall incidence of secondary neoplasms of the larynx is rare accounting for 0.09 to $0.4 \%$ [5]. Adenocarcinoma deposits in laryngeal area are barely known. Its frequency increases with age (median 58 years, range 24-83 years) and it tends to occur more commonly in males by a ratio of $2: 1[1,2]$.

The signs and symptoms are similar to those produced by primary laryngeal tumours but the severity of presentation is dependent on the location of the tumour deposit. In our case the lesion was mostly within the cricoid cartilage with sclerotic expansion of the cartilage and consequent narrowing of the subglottis which triggered respiratory distress. The prognosis is generally poor as the condition is often associated with terminal widespread disseminated disease.

Our case describes a rare case of laryngeal metastasis of colon adenocarcinoma. A similar case was last described in 1997 by Puxeddu and others [5] who reported a case of colon adenocarcinoma with massive transglottic tumour metastasis obstructing the airway. However, the uniqueness of our case is the vagueness of both endoscopic examination and radiological findings that are inconsistent with malignancy. A lack of classical changes of the larynx typical of malignant conditions was problematic in this case in arriving at a diagnosis but the possibility of metastasis as well as primary non-squamous neoplasms which are typically submucosal should be contemplated. It can be only assumed how many underdiagnosed patients with laryngeal cartilages thickening on radiological report could have been diagnosed with secondary laryngeal neoplasms and possibly treated with a different approach. Thus, it is vitally important to be aware of rare locations for metastasis within the larynx and insisting on multiple site tissue biopsies for a diagnosis.

\section{CONCLUSION}

- Laryngeal metastases are rare in general due to the anatomy of lymphocirculatory system of larynx and are especially uncommon for cancer originating from colon.

- The presented case describes a unique clinical picture with a laryngeal metastasis mimicking extensive cricoid sclerosis. Such presentation has never been described before.

- Metastasis to the larynx and some non-squamous primary neoplasms are typically subepithelial.

- Late recognition of atypical presentation of laryngeal metastases can negatively affect patient's prognosis.

\section{CONFLICT OF INTEREST}

Declared None.

\section{ACKNOWLEDGEMENTS}

Declared None.

\section{REFERENCES}

[1] Batsakis JG, Luna MA, Byers RM. Metastases to the larynx. Head Neck Surg 1985; 7: 458-60.

DOI:10.1002/hed.2890070604

[2] Ferlito A. Secondary neoplasms. In: Ferlito A, Ed. Neoplasms of the Larynx. Edinburgh: Churchill Livingston 1993; pp. $349-60$.

[3] El-Naggar AK. Secondary neoplasms of the larynx. In: Fried MP, Ferlito A, Eds. The larynx. $3^{\text {rd }}$ ed. San Diego: Pleural Publishing 2009; vol. II: pp. 719-28.

[4] Ferlito A, Caruso G, Recher G. Secondary laryngeal tumours. Report of seven cases with review of the literature. Arch Otolaryngol Head Neck Surg 1988; 114: 635-9.

DOI:10.1001/archotol.1988.01860180049028

[5] Puxeddu R, Pelagatti CL, Ambu R. Colon adenocarcinoma metastatic to the larynx. Eur Arch Otorhinolaryngol 1997; 254(7): 353-5. DOI:10.1007/BF02630729 